\title{
Characterization of Pakistani Wheat Germplasm for High and Low Molecular Weight Glutenin Subunits Using SDS-PAGE
}

\author{
A. KHALID ${ }^{1,2 *}$ and A. HAMEED ${ }^{1,2}$ \\ ${ }^{1}$ Nuclear Institute for Agriculture and Biology (NIAB), Jhang Road, Faisalabad, Pakistan \\ ${ }^{2}$ Pakistan Institute of Engineering and Applied Sciences, Islamabad, Pakistan \\ (Received 17 September 2018; Accepted 25 February 2019; \\ Communicated by F. Békés)
}

\begin{abstract}
High molecular weight (HMW-GS) and low molecular weight (LMW-GS) glutenin subunits play a significant role in bread making quality and extensibility, though they signify merely $10 \%$ and $40 \%$ of the entire seed storage proteins. For the estimation of bread quality on the basis of allelic difference in HMW-GS and LMW-GS at Glu-1 and 3 loci, wheat germplasm (77 genotypes) was collected from diverse agro-climatic regions of Pakistan and characterized by using sodium dodecyl sulphate polyacrylamide gel electrophoresis (SDSPAGE). Thirty distinct allelic arrangements were identified with a sum of thirteen Glu-1 alleles. Maximum frequency of allele 1 was found in twenty-nine genotypes at Glu-A1 locus while high proportion of subunit pairs $13+16$ and $2+12$ was detected in 33 and 32 genotypes at Glu-B1 as well as Glu-D1 locus, respectively. Few rare alleles were also separated out. The quality scores ranged from 4-10, however highest quality score of ten was more recurrent (36.36\%). A good quality score of 8 and 6 were found in $32.47 \%$ as well as $19.48 \%$ of genotypes individually. In LMW-GS, seventeen diverse combinations of alleles with aggregate of ten Glu-3 alleles were detected. Glu-A3c and Glu-B3d alleles were observed in $33(42.85 \%)$ genotypes, encoding high sedimentation and protein contents. Hence, this will enable the breeders to utilize both glutenin subunits as biochemical indicator for selecting superior wheat genotypes possessing enhanced bread making quality.
\end{abstract}

Keywords: wheat, bread making quality, HMW-GS, LMW-GS, SDS-PAGE

\section{Introduction}

Wheat is the principal cereal crop globally with respect to utilization as well production. It is the main source of protein, dietary fibre and energy in human food. In Pakistan, it is the basic food which fulfils over sixty per cent of the overall necessities for protein and calories in normal daily life and is consumed in various forms, primarily as bread, chapatti, pizzas, macaroni, doughnuts, spaghetti, noodles and cakes. Mature grains of wheat constitute proteins, i.e. 8-20\% (Anjum et al. 2007). Its complex genetic background is responsible for enormous diversity in processing and nutritional qualities among cultivars (Jang et al. 2017).

\footnotetext{
*Corresponding author; E-mail: invincible_me2@yahoo.com
} 
Bread making quality of wheat is primarily influenced by its protein content and protein quality. Gluten proteins determine the wheat flour ability to be processed into variety of foods (Zorica Jurkovic et al. 2000). It makes up to $80-85 \%$ of total flour protein and impart characteristics of extensibility and elasticity which are indispensable for wheat flours functionality and hence specify bread making quality (Costa et al. 2013). The suitable equilibrium between these two attributes makes wheat gluten acceptable for bread making. Elasticity is defined as stretching capability determination when dough is subjected to a force. On the other hand, extensibility involves stretching in the direction to which force is applied (Jang et al. 2017).

The gliadins and glutenins encompass each about fifty per cent of gluten proteins (Anjum et al. 2007). On the basis of their molecular weight two types of glutenin subunits have been differentiated into high molecular weight glutenin subunit, HMWGSs $(97-136 \mathrm{kDa})$ and low molecular weight subunits, LMWGSs (31-48 kDa) (Zorica Jurkovic 2000). Wheat endosperm comprises of high molecular weight glutenin subunits (HMWGSs), which are primary grain storage protein comprises of only almost $12 \%$ of the total protein and has a crucial role in wheat gluten as a skeletal network (Geng et al. 2014). LMW-GS signify almost $40 \%$ of wheat gluten and is responsible for defining dough resistance plus extensibility (Park et al. 2011).

Studies have revealed that the HMW-GS have huge impact on the dough's rheological characteristics and bread forming attribute (Peña et al. 2005; Li et al. 2010; Zhang et al. 2011; Hernandez et al. 2012) and LMW-GS from wheat affect bread and noodle processing quality (Lee et al. 2017). Rheological as well as bread manufacturing experiments are arduous and need time, large amount of samples. So, the flour attributes identification through fast and precise methods for all kind of product permits cultivars choice with suitable traits for industrial usage in a smaller amount of time, certifying adequacy and success of fresh cultivar (Jang et al. 2017).

Sodium dodecyl sulphate poly acrylamide gel electrophoresis (SDS-PAGE) test is a classical technique employed for protein components separation. It enables the segregation of the gluten proteins subunits by identifying high molecular weight (HMW-GS) and low molecular weight (LMW-GS) glutenin subunits individually. Genes which encode high molecular glutenin subunits are situated at Glu-A1, Glu-B1 and Glu-D1 position on the long arms of A1, B1, and D1 chromosomes, correspondingly (Payne et al. 1984), while those who encode the low molecular glutenin subunits are present at Glu-A3, Glu-B3 and Glu-D3 position on the short arms of A1, B1, and D1 chromosomes, correspondingly. Every locus possesses two strongly associated paralogous genes coding two diverse forms of HMW-GS, viz. $x$ and y type subunits (Jang et al. 2017).

Hence, in current study, aim is to characterize the allelic differences among cultivars through SDS-PAGE and assessment of their association with end-use quality to upgrade the quality of available germplasm which will enable the breeders to develop wheat cultivars using specific HMW-GS, LMW-GS alleles with sufficient gluten stability and resilience for bread production. 


\section{Material and Methods}

\section{Plant material collection}

Wheat germplasm of 77 varieties were gathered from different centres within every single agroclimatic region of Pakistan (Khalid and Hameed 2017), in order to evaluate of should be omitted high molecular weight glutenin subunits. Reference varieties namely, Gabo (2, $\left.2^{*}, 12,17,18\right)$, Holdfast $(1,5,7,8,11)$ and Chinese-Spring $(2,7,8,12)$ were employed as references (Payne and Lawrence 1983) while Cheyenne (c, e, f), Chinese Spring (a, a, a), Gabo (b, b, b), Glenlea (g, g, c), Insignia (f, c, c), Marquis (e, b, a), Nanbu-Komugi (d, ab, a), Norin 61 (d, i, c), Orca (d, d, c), Opata (b, ad, a) and Suneca (d, h, e) were used as standard for low molecular weight glutenin subunits (Gupta and Shepherd 1990).

\section{Seed Protein Extraction}

For total proteins extraction, five single grains of each genotype were crushed in $50 \mathrm{mM}$ phosphate buffer ( $\mathrm{pH}$ 7.8) then centrifuged in micro-centrifuge machine (Sigma 1-14) for ten minutes at 14,000 rpm. Dye binding assay was employed to measure amount of protein extracts as defined by (Bradford 1976). Supernatant was combined (4:1) with cracking solution (10 $\mathrm{ml}$ having $1 \mathrm{~g}$ SDS, $0.01 \mathrm{~g}$ bromphenol blue, $2 \mathrm{ml}$ Mercaptoethanol, 1.5 $\mathrm{ml} 0.5 \mathrm{M}$ tris, $\mathrm{pH} 6.8,5 \mathrm{~g}$ sucrose and $6.5 \mathrm{ml}$ water) and mixed on vortex mixer, heated in a hot water bath ( $5 \mathrm{~min}$ ) for proteins denaturation.

\section{Seed Protein Profiling}

Sodium dodecyl sulphate-polyacrylamide gels electrophoresis was used to perform samples protein profiling as defined by Laemmli (1970). Same amount of samples were laden into ten per cent gels. Continuous voltage (180 and 160 volts) was applied to conduct electrophoresis. Eventually, gels were fixed in solution comprising $10 \%$ acetic acid and $40 \%$ ethanol for fifteen minutes and agitated on a shaker persistently. After fixation, gel was washed with distilled water for fifteen minutes with altering water after every five minutes. Gels were then stained with coomassie blue G-250 dye and de stained in water overnight.

\section{Gel Documentation and Analysis}

Eventually, gels were photographed via UVIpro platinum gel documentation system (UVItec UK).

\section{Quality Scoring}

The HMW-glutenin subunits were recognized through formerly suggested system of numbering (Payne and Lawrence 1983) whereas quality scores were given by scoring system defined by (Payne 1987). Alleles were consigned quality scores of 1 (low quality) 
to 4 (high quality) and separate scores for all allele existing in a genotype were combined to render the total quality score for respective genotype.

\section{Statistical Analysis}

The produced data matrix was then statistically analyzed using computer software Microsoft Excel along with XLSTAT Version 2012.1.02, Copyright Addinsoft 1995-2012 (http://www.xlstat.com).

\section{Results}

The outcomes revealed high polymorphism level regarding high molecular weight-glutenin subunits (Fig. S1). Amongst the 77 tested genotypes, 74 were homogeneous $(96.10 \%)$ while three were heterogeneous $(3.89 \%)$ with respect to high molecular weightglutenin subunits. Thirty distinct combinations of alleles were identified (Table $\mathrm{S} 1^{*}$ ). Overall, thirteen Glu-1 alleles, three at Glu-A1 locus, six at Glu-B1 locus while four at Glu-D1 locus were found in estimated material. Three alleles $(1,2 *$ and Null) were detected at A genome's Glu-1A locus (Table S1). Occurrence rate of two active high molecular weight-glutenin subunits i.e. $1,2 *$ besides one null form of gene holding no subunit were $37.66 \%, 35.06 \%$ and $26 \%$, respectively (Fig. S2a). Variation was found maximum for Glu-B1 locus in comparison with Glu-A1 besides Glu-D1. Alleles analogous to 6 diverse forms of subunits, 4 in subunit pairs viz., $13+16,17+18,7+9,7+8$ and 2 single/rare Glu-B1 subunits such as 7 and 20 in lesser proportions were sorted out in entire genotypes correspondingly (Table S1). Rate recorded for prevalent loci $13+16$ besides $17+18$ were $42.85 \%$ and $28.57 \%$ correspondingly (Fig. S2b). Glu-D1 locus displayed alleles responsible for subunits pair $5+10$ and $2+12$ as a prevailing combination with occurrences $55.84 \%$ in addition to $41.55 \%$ respectively. The existence of single subunits viz. 10 with low frequencies and a subunit combination $2+10(3.89 \%)$ were also detected (Fig. S2c).

Results elaboration for allelic difference quantification in genomes of wheat exhibited highest input of genome B (46\%) pursued by genome (31\%) as shown in Fig. S3.

Afterwards, distinct scores of high molecular weight-glutenin subunit were combined to determine genotype's quality score in line with Payne et al. (1987) as shown in Table S1. The outcomes of quality score on the basis of high molecular weight-glutenin subunit composition amongst varieties and advance lines showed larger alteration with advance lines normally displaying a low score. The score ranged from 4-10 having a mean of 7. Twenty-eight genotypes attained the maximum quality score of $10(32.47 \%)$ and 24 had a good quality score of 9 (5.19\%). Generally, a good quality score of 8 was much persistent (39.47\%) in current analysis. Average quality score of 7 was stable in one genotype merely. Further poor quality scores viz, 6, 5 and 4 seemed in comparatively lower frequencies with $19.48 \%, 1.30 \%$ and $2.60 \%$, respectively (Fig. S4).

*Further details about the Electronic Supplementary Material (ESM) can be found at the end of the article. 
In LMW-GS, fourteen different allelic combinations were detected (Table S2). A total of 10 Glu-3 alleles (3 at Glu-A3 locus, 4 at Glu-B3 locus and 4 at Glu-D3 locus) were identified in estimated material. At Glu-3A locus, three alleles, i.e. c, d and e were detected (Table S2) among which Glu-A3c was found in $42.85 \%$ of the genotypes (Fig. S5a). There was more variation for Glu-B3 locus as compared to Glu-A3 and GluD3. Out of four alleles (b, d, h, i), d and h alleles were predominant and revealed a high frequency of $42.85 \%$ and $23.37 \%$, respectively (Fig. S5b), while allele b and i in lower frequencies $11.68 \%$ and $22 \%$ were also found in all the genotypes (Table S2). At Glu-D3 locus, three alleles were detected having alleles $\mathrm{a}, \mathrm{b}$ and $\mathrm{c}$ where allele a holds a dominant frequency of $77.92 \%$ and the existence of allele $b$ and $\mathrm{c}$ with low frequencies of $9.09 \%$ and $12.98 \%$ were also observed (Fig. S5c).

\section{Discussion}

Wheat glutenin proteins, especially high molecular weight and low molecular weight glutenin subunits accumulated in starchy endosperm have a vital part in defining the bread-making as well as dough characteristics and hence the nutritional quality of flour (Anjum et al. 2007; Peng et al. 2016). According to the research, HMW-glutenin subunits composition variation may be the reason behind rate of inter varietal differences in bread forming capability while LMW-GS have not attained that much attention as HMW-GS because of intricate banding pattern and mobility overlapping with glaidin (Park et al. 2011), so this makes it necessary to evaluate the variation in alleles of high molecular weight as well as low molecular weight glutenin subunits conformation liable for bread manufacturing quality amongst wheat varieties of Pakistan (Tabasum et al. 2011).

In current study, HMW-glutenin subunits polymorphism was observed among Pakistani wheat varieties and has also been stated in the previous studies (Yan et al. 2007; Chaparzadeh et al. 2008). Homogeneous and heterogeneous arrays of protein were detected in our genotypes concurring with the former studies (Popa et al. 2003; Kang et al. 2007; Singh et al. 2007). This heterogeneous state can be utilized in breeding strategies for enhancing bread forming quality through selecting a glutenin physical trait having high molecular weight-glutenin subunit configuration related to good quality. Thirty diverse combination of alleles with aggregate of thirteen Glu-1 alleles illustrated not merely occurrence of allelic difference in HMW-glutenin subunits, accountable for alterations in bread manufacturing attributes nevertheless its possible application for breeding varieties in future.

Storage protein conformation is thought as constant element of cultivar due to direct genome expression therefore; it can help in identification of cultivar. Our results are in accordance with the earlier studies of (Popa et al. 2003; Kang et al. 2007; Tsenov et al. 2009).

In present research, three alleles, i.e. $1,2 *$ and Null were contributed by Glu-A1 locus of A genome with the prevalence of 1 subunit (37.7\%) followed by subunit $2 *(35.1)$ and Null $(26 \%)$. In contrast to present findings, subunit $2 *$ was found predominant with a percentage of $72.09 \%$ and 25\% previously (Bahraei et al. 2004; Chaparzadeh et al. 2008) 
while (Chaparzadeh et al. 2008) reported lack of subunit one in their genotypes. These variations might be the result of germplasm differences. The subunit 1 has good influence on the wheat quality; thus it may be introgressed into the genetic contextual of native varieties to enhance their quality followed by sustained breeding attempts as well as selection procedures (Tabasum et al. 2011; Costa et al. 2013).

Results obtained in this study coincide with findings of (Morgunov et al. 1990) who assessed a small number of Null alleles in Soviet wheat genotypes. Though, Nakamura (2000) reported high frequency of null allele i.e. $80.4 \%$ and $74.1 \%$ in Chinese and Japanese wheat varieties while highest frequency of $48.3 \%$ was reported by Popa et al. (2003) in Romanian wheat varieties whereas frequency of Null allele was found dominant in French wheat i.e. $69.5 \%$ by Branlard et al. (2003).

Glu-B1 of genome B depicted greater polymorphism level by rendering 6 diverse kinds of allelic alternates. Formerly, highest glutenin polymorphism at Glu-B1 locus has also been stated (Yan et al. 2007). The greatest recurrent pattern associated with good bread making quality was of subunits $13+16(42.85 \%)$ followed by $17+18(28.57 \%)$. Formerly, lower frequencies $(3.57 \%)$ of $13+16$ subunits were reported in Iranian wheat cultivars and advanced breeding lines (Mandoulakani et al. 2006) whereas in another study, much lower frequency of $0.7 \%$ and $0 \%$ in Chinese as well as Japanese wheat varieties (Nakamura 2000) were found. Yet in another study low frequency of about 5\% was detected in Pakistani wheat germplasm (Tabasum et al. 2011). However, subunits $17+18$ were most frequently encountered in hexaploid wheat landraces of Pakistan (Tahir et al. 1996). In another finding, the highest frequencies were observed for subunits $7+9(82.6 \%)$ (Popa et al. 2003) still another finding reported the highest frequencies $(29 \%)$ and $(91 \%)$ of subunits $7+8$ in European wheat landraces and obsolete cultivars (Gregová et al. 2006) as well as in Hand-Extended Noodles made from wheat flour (Kang et al. 2007).

Single/rare subunits of 7 and 20 were found similarly in our particular genotypes. Sub unit 20 screened out in nine varieties $(11.68 \%)$ is in accordance with the results of Yan et al. (2007) who had stated little proportion in their genotypes regarding above mentioned subunit. Contrary to our findings, Carrillo et al. (1995) reported subunit 20 as most recurrent Glu-B1 allele. These subunits scarcity is beneficial because of their poor bread-making quality (Payne 1987; Dong et al. 2009).

Glu-D1 locus of genome D revealed substantial amount of polymorphism as well. The alleles holding the subunits pairs $5+10$ as well as $2+12$ were the highest persistent patterns with a frequency of $55.8 \%$ and $41.6 \%$. Subunit pairs $5+10$ impart beneficial effect on bread making quality (Costa et al. 2013). Our results are analogous to the findings of (Nakamura 2000; Popa et al. 2003; Kang et al. 2007) where examined varieties possessed leading frequencies of $5+10$ and $2+12$ subunits in numerous collections. In contrast to our results, (Branlard et al. 2003; Chaparzadeh et al. 2008) stated a lower frequency of subunit $5+10$. One genotype in present study had $2+10$ subunit whose quality contribution is not known and is in accordance with the findings of Yan et al. (2007).

Various genes in species genome are responsible for seed proteins and their study can offer valuable information regarding evolutionary associations and genome complexity 
in species (Schuster et al. 1997; Tabasum et al. 2011). The division of allelic difference into genomic involvement showed that genome $\mathrm{B}$ is the basis of genetic diversity though highest contribution towards quality is via genome $\mathrm{D}$.

Bread manufacturing aspect is a composite characteristics swayed through environment. Though, evaluation of bread-making quality potential is possible with the help of HMW-glutenin subunits in wheat genotypes (Schuster et al. 1997). Highest and good quality score of 10, 8 and 6 was found in tested genotypes. On the whole, our germplasm holds a good bread making potential as only one variety and 2 advance lines were detected to enclose a score of 5 or less. Our results varied from Schuster et al. (1997) who proposed mostly poor bread making quality in tested Brazilian wheat genotypes. Genotypes with lower frequency of good quality score specify the varieties development without utilising the comprehensive information of glutenin subunits conformation besides their role in bread making quality.

In present research, seventeen diverse combination of alleles with aggregate of ten Glu-3 alleles illustrated occurrence of allelic difference in LMW-glutenin subunits, accountable for alterations in bread and dough manufacturing attributes. Three alleles i.e. c, $\mathrm{d}$ and e were contributed by Glu-A3 locus of A genome. Allele GluA3c was found predominant with a frequency of $42.85 \%$. Contrary to our findings, Park et al. (2011) reported maximum frequency of Glu-A3d in genotypes of Korean wheat while the same frequency was also detected in Japanese and French wheat (Branlard et al. 2001; Tanaka et al. 2005). However, our results are analogous to the previous findings (Branlard et al. 2003; Bellil et al. 2010; Bradová and Štočková 2010; Liu et al. 2010; Ram et al. 2011; Peng et al. 2016) where tested varieties possessed leading frequency of Glu-A3c allele i.e. $49.3 \%, 47.0 \%, 44.5 \%, 72.5 \%, 40.3 \%, 64.6 \%$ respectively. So, these results showed that the alleles Glu-A3c holds predominance throughout the world with respect to bread wheat. It has found to have high sedimentation volume so it can play vital role in quality improvement (Khan 1984). Formerly, He et al. (2005) reported the Glu-A3d as desirable allele for gluten quality and pan bread quality and it was also found in our evaluated material with a frequency of $35.06 \%$.

Lowest frequency of $22.07 \%$ in Glu-A3e was found in present study. This non desirable Glu-A3e allele which is primary cause of reduction in maximum resistance and extensibility of dough (Appelbee 2007) was also found least frequent (3.5\%) in Chinese wheat landraces, suggesting the high value of wheat varieties for modern wheat quality improvement.

For LMW-GS, the Glu-3 alleles have been already ranked according to their $\mathrm{R}_{\max }$ (maximum dough resistance) (Henkrar et al. 2017). In the tested genotypes, Glu-A3c allele represented $42.85 \%$, and as stated by $\mathrm{R}_{\max }$, this c allele is associated with least dough resistance and poor quality. Previously, majority of allele $\mathrm{c}$ has been reported at Glu-A3 locus in Argentinean (Lerner et al. 2009), Morrocan (Henkrar et al. 2017) and US (Shan et al. 2007) bread wheat cultivars.

The predominant alleles in our study were Glu-B3d with a frequency of $42.85 \%$. Our results coincide with the previous findings (Cornish et al. 1993; Luo et al. 2001; Bellil et al. 2010; Henkrar et al. 2017) who reported maximum frequency of d allele in Saharan 
bread wheat cultivars and French cultivars but lowest frequency of Glu-B3b. For the GluA3 and Glu-B3, our results differ from Aguiriano et al. (2008) who reported the prevalence of allele a for both locus in Spanish durum landraces. Glu-B3d has been reported to have Superior mixograph characteristics, high sedimentation and strong gluten strength (Branlard et al. 2001; Khan et al. 2016) and hence can be pooled by breeders for quality improvement.

In present research, three alleles i.e. $\mathrm{a}, \mathrm{b}$ and $\mathrm{c}$ were contributed by Glu-D3 locus of D genome with the prevalence of allele a $(77.92 \%)$ followed by allele c $(12.98 \%)$ and allele b $(9.09 \%)$. Our findings regarding Glu-D3 allele are in accordance with frequencies previously found where Liu (2008) reported maximum occurrence of Glu-D3a in 233 Chinese bread wheat while differ from the findings of Shan et al. (2007) and Lerner et al. (2009) with Glu-D3b as a major allele. Branlard et al. (2001) reported that allele GluB3a has positive impact on strength of dough. Hence, Glu-A3 and Glu-B3 loci alleles of LMW-GS are noteworthy because of their great influence on wheat processing qualities and in turn gluten elasticity. These alleles are accountable for high protein contents and sedimentation, which defines their important role in wheat quality improvement (Khan et al. 2016).

Wheat germplasm collected from diverse agro-climatic regions of Pakistan was tested in this study. It was major factor responsible for the difference in genetic makeup and for being genetically diverse and is in turn responsible for the variation in distribution of genes/alleles. Difference in allelic variation causes distinct HMW-GS and LMW-GS combinations. So, as the mutual occurrence of diverse alleles from the three loci is significant in terms of scores accumulation as well as quality determination, the assessment of genetic variance concerning loci encoded glutenin proteins, becomes important to characterize existing germplasm for their utilization.

Recent study defined the allelic variants of HMW-glutenin subunits which revealed strong association with high bread making quality as twenty-nine genotypes showed highest frequency of allele 1 at Glu-A1 locus whereas 33 and 32 genotypes revealed high proportion of subunit pairs $13+16$ and $2+12$ at Glu-B1 as well as Glu-D1 locus, respectively. Twenty-eight genotypes exhibited maximum quality score of 10 while Glu-A3c and GluB3d alleles of LMW-GS were also found significant with respect to bread processing quality. Based on these novel findings, genotypes having good quality subunits can be pooled by breeders through diverse breeding techniques so as to develop high quality wheat in future.

\section{Acknowledgements}

Authors are thankful for provision of facilities and help in analysis at the Nuclear Institute for Agriculture and Biology MAB-Lab1, Faisalabad. The paper is part of $\mathrm{PhD}$ thesis of first author i.e. Anam Khalid. 


\section{References}

Aguiriano, E., Ruiz, M., Fite, R., Carrillo, J.M. 2008. Genetic variation for glutenin and gliadins associated with quality in durum wheat (Triticum turgidum L. ssp. turgidum) landraces from Spain. Span. J. Agric. Res. 6:599-609.

Anjum, F.M., Khan, M.R., Din, A., Saeed, M., Pasha, I., Arshad, M.U. 2007. Wheat gluten: high molecular weight glutenin subunits structure, genetics, and relation to dough elasticity. J. Food Sci. 72:56-63.

Appelbee, M. J. 2007. Quality potential of gluten proteins in hexaploid wheat and related species. PhD Thesis, School of Agriculture, Food and Wine, The University of Adelaide, SA, Australia.

Bahraei, S., Saidi, A., Alizadeh, D. 2004. High molecular weight glutenin subunits of current bread wheats grown in Iran. Euphytica 137:173-179.

Bellil, I., Bouguennec, A., Khelifi, D. 2010. Diversity of seven glutenin and secalin loci within triticale cultivars grown in France. Not. Bot. Horti Agrobo. 38:48-55.

Bradford, M.M. 1976. A rapid and sensitive method for the quantitation of microgram quantities of protein utilizing the principle of protein-dye binding. Anal. Biochem. 72:248-254.

Bradová, J., Stockova, L. 2010. Evaluation of winter wheat collection in terms of HMW- and LMW-glutenin subunits. Czech Journal of Geneicst and Plant Breeding 46:96-99.

Branlard, G., Dardevet, M., Saccomano, R., Lagoutte, F., Gourdon, J. 2001. Genetic diversity of wheat storage proteins and bread wheat quality. Euphytica 119:59-67.

Branlard, G., Dardevet, M., Amiour, N., Igrejas, G. 2003. Allelic diversity of HMW and LMW glutenin subunits and omega-gliadins in French bread wheat (Triticum aestivum L.). Genet. Resour. Crop Ev. 50:669679.

Carrillo, J., Vázquez, J., Ruiz, M. 1995. Variability for glutenin proteins in Spanish durum wheat landraces. Options Mediterannennes. Serie A. Seminaires Mediterraneens: 143-147.

Chaparzadeh, N., Sofalian, O., Javanmard, A., Hejazi, M., Zarandi, L. 2008. Study of glutenin subunits in some wheat landraces from northwest of Iran by SDS-PAGE technique. Int. J. Agric. Biol. 10:101-104.

Cornish, G., Burridge, P., Palmer, G., Wrigley, C. 1993. Mapping the origins of some HMW and LMW glutenin subunit alleles in Australian wheat germplasm In: Proceedings of the 43rd Australian Cereal Chemistry Conference, C.W. Wrigley (ed.), Guthrie Centenary Conference. pp. 225-260.

Costa, M.S., Scholz, M.B.D.S., Franco, C.M.L. 2013. Effect of high and low molecular weight glutenin subunits, and subunits of gliadin on physicochemical parameters of different wheat genotypes. Food Sci. Technol. (Campinas) 33:163-170.

Dong, K., Hao, C., Wang, A., Cai, M., Yan, Y. 2009. Characterization of HMW glutenin subunits in bread and tetraploid wheats by reversed-phase high-performance liquid chromatography. Cereal Res. Commun. 37:65-73.

Geng, Y., Pang, B., Hao, C., Tang, S., Zhang, X., Li, T. 2014. Expression of wheat high molecular weight glutenin subunit $1 \mathrm{Bx}$ is affected by large insertions and deletions located in the upstream flanking sequences. PloS one 9:105363.

Gregová, E., Hermuth, J., Kraic, J., Dotlacil, L. 2006. Protein heterogeneity in European wheat landraces and obsolete cultivars: Additional information II. Genetic Resour. Crop Ev. 53:867-871.

Gupta, R.B., Shepherd, K. 1990. Two-step one-dimensional SDS-PAGE analysis of LMW subunits of glutelin. Theor. Appl. Genet. 80:65-74.

He, Z.H., Liu, L., Xia, X.C., Liu, J.J., Pena, R. 2005. Composition of HMW and LMW glutenin subunits and their effects on dough properties, pan bread, and noodle quality of Chinese bread wheats. Cereal Chem. 82:345-350.

Henkrar, F., El-Haddoury, J., Iraqi, D., Bendaou, N., Udupa, S.M. 2017. Allelic variation at high-molecular weight and low-molecular weight glutenin subunit genes in Moroccan bread wheat and durum wheat cultivars. Biotechnology 7:287.

Hernandez, Z., Figueroa, J., Rayas-Duarte, P., Martínez-Flores, H., Arambula, G., Luna, G., Pena, R. 2012. Influence of high and low molecular weight glutenins on stress relaxation of wheat kernels and the relation to sedimentation and rheological properties. J. Cereal Sci. 55:344-350. 
Jang, Y.R., Beom, H. R., Altenbach, S.B., Lee, M.K., Lim, S.H., Lee, J.Y. 2017. Improved method for reliable HMW-GS identification by RP-HPLC and SDS-PAGE in common wheat cultivars. Molecules 22:1055.

Kang, Z.Y., Wang, J.J., Shang, X.W. 2007. Score system study for hand-extended noodle quality based on HMW-GS index in wheat flour. Agr. Sci. China 6:304-310.

Khalid, A., Hameed, A. 2017. Seed biochemical analysis based profiling of diverse wheat genetic resource from Pakistan. Front. Plant Sci. 8:1-15.

Khan, M. 1984. Nutritional attributes of wheat. Progressive Farming 4:23-26.

Khan, T.N., Akram, Z., Ajmal, S.U.K., Naqvi, S., Mujeeb, A. 2016. GLU-A3 and GLU-B3 alles for LMW protein in local wheat identification of GLU-A3 and GLU-B3 alleles for LMW Protein Subunits in some wheat genotypes of Pakistan. Pak. J. Agr. Sci. 53:495-500.

Laemmli, U.K. 1970. Cleavage of structural proteins during the assembly of the head of bacteriophage T4. Nature 227:680-685.

Lee, J.-Y., Jang, Y.-R., Beom, H.-R., Altenbach, S.B., Lim, S.-H., Lee, C.K. 2017. Allelic analysis of low molecular weight glutenin subunits using 2-DGE in Korean wheat cultivars. Breeding Sci. 67:398-407.

Lerner, S., Kolman, M., Rogers, W. 2009. Quality and endosperm storage protein variation in Argentinean grown bread wheat. I. Allelic diversity and discrimination between cultivars. J. Cereal Sci. 49:337-345.

Li, Y., Zhou, R., Branlard, G., Jia, J. 2010. Development of introgression lines with 18 alleles of glutenin subunits and evaluation of the effects of various alleles on quality related traits in wheat (Triticum aestivum L.). J. Cereal Sci. 51:127-133.

Liu, L., Ikeda, T.M., Branlard, G., Peña, R.J., Rogers, W.J., Lerner, S.E., Kolman, M.A., Xia, X., Wang, L., Ma, W. 2010. Comparison of low molecular weight glutenin subunits identified by SDS-PAGE, 2-DE, MALDITOF-MS and PCR in common wheat. BMC Plant Biol. 10:124.

Luo, C., Griffin, W., Branlard, G., Mcneil, D. 2001. Comparison of low- and high molecular-weight wheat glutenin allele effects on flour quality. Theor. Appl. Genet. 102:1088-1098.

Mandoulakani, B., Gomarian, M., Shahnejat-Bushehri, A. 2006. Identification of the parents for bread-making quality improvement in bread wheat based on RAPD and seed storage protein (HMW-GS) markers. Pak. J. Biol. Sci. 9:497-502.

Morgunov, A., Rogers, W., Sayers, E., Metakovsky, E. 1990. The high-molecular-weight glutenin subunit composition of Soviet wheat varieties. Euphytica 51:41-52.

Nakamura, H. 2000. Allelic variation at high-molecular-weight glutenin subunit Loci, Glu-A1, Glu-B1 and Glu-D1, in Japanese and Chinese hexaploid wheats. Euphytica 112:187.

Park, C.S., Kang, C.S., Jeung, J.U., Woo, S.H. 2011. Influence of allelic variations in glutenin on the quality of pan bread and white salted noodles made from Korean wheat cultivars. Euphytica 180:235-250.

Payne, P., Holt, L.M., Jackson, E.A., Law, C., Damania, A. 1984. Wheat storage proteins: their genetics and their potential for manipulation by plant breeding. Philosophical Transactions of the Royal Society of London. Series B, Biological Sciences. pp. 359-371.

Payne, P.I., Lawrence, G.J. 1983. Catalogue of alleles for the complex gene loci, Glu-A1, Glu-B1, and Glu-D1 which code for high-molecular-weight subunits of glutenin in hexaploid wheat. Cereal Res. Commun. 11:29-35.

Payne, P.I. 1987. Genetics of wheat storage proteins and the effect of allelic variation on bread-making quality. Ann. Rev. Plant Physio. 38:141-153.

Peña, E., Bernardo, A., Soler, C., Jouve, N. 2005. Relationship between common wheat (Triticum aestivum L.) gluten proteins and dough rheological properties. Euphytica 143:169-177.

Peng, Y., Yu, Z., Islam, S., Zhang, Y., Wang, X., Lei, Z., Yu, K., Sun, D., Ma, W. 2016. Allelic variation of LMW-GS composition in Chinese wheat landraces of the Yangtze-River region detected by MALDI-TOFMS. Breeding Sci. 66:646-652.

Popa, M., Gregova, E., Kraic, J. 2003. Romanian wheat (Triticum aestivum L.) landraces characterized by seed storage protein electrophoresis. Plant Genetic Resources Newsletter (IPGRI/FAO), Bulletin des Ressources Phytogenetiques (IPGRI/FAO) Noticiario de Recursos Fitogeneticos (IPGRI/FAO).

Ram, S., Sharma, S., Verma, A., Tyagi, B.S., Pena, R.J. 2011. Comparative analyses of LMW glutenin alleles in bread wheat using allele-specific PCR and SDS-PAGE. J. Cereal Sci. 54:488-493. 
Schuster, I., Souza, M.a.D., Cardoso, A.A., Sediyama, C.S., Moreira, M.A. 1997. Correlation between high molecular weight gluten subunits composition and bread-making quality in Brazilian wheat. Braz. J. Genet. 20:11-25.

Shan, X., Clayshulte, S.R., Haley, S.D., Byrne, P.F. 2007. Variation for glutenin and waxy alleles in the US hard winter wheat germplasm. Journal of Cereal Science, 45:199-208.

Singh, A., Deveshwar, J., Ahlawat, A., Singh, B. 2007. Identification of novel variants of high molecular weight glutenin subunits in Indian bread wheat landraces. Cereal Res. Commun. 35:99-108.

Tabasum, A., Iqbal, N., Hameed, A., Arshad, R. 2011. Evaluation of Pakistani wheat germplasm for bread quality based on allelic variation in HMW glutenin subunits. Pak. J. Bot. 43:1735-1740.

Tahir, M., Turchetta, T., Anwar, R., Lafiandra, D. 1996. Assessment of genetic variability in hexaploid wheat landraces of Pakistan based on polymorphism for HMW glutenin subunits. Genet. Resour. Crop Ev. 43:211-220.

Tanaka, H., Toyoda, S., Tsujimoto, H. 2005. Diversity of low-molecular-weight glutenin subunit genes in Asian common wheat (Triticum aestivum L.). Breeding Science, 55:349-354.

Tsenov, N., Atanasova, D., Todorov, I., Ivanova, I., Stoeva, I. 2009. Allelic diversity in Bulgarian winter wheat varieties based on polymorphism of glutenin subunit composition. Cereal Research Communications, 37:551-558.

Yan, Z.H., Dai, S.F., Liu, D.C., Wei, Y.M., Zheng, Y.L. 2007. Allelic variation of high molecular weight glutenin subunits in the hexaploid wheat landraces of Tibet, China. International Journal of Agriculture and Research, 2:838-843.

Zhang, C., Dong, C., He, X., Zhang, L., Xia, X., He, Z. 2011. Allelic variants at the locus on wheat chromosome 2DL and their association with yellow pigment content. Crop Science, 51:1580-1590.

Zorica Jurkovic, R., Georg Drezner, Danieia Hervat. 2000. The HMW gluenin subunit composition of OS wheat cultivars and their relationship with bread-making quality. Cereal Research Communication, 28:271277.

\section{Electronic Supplementary Material (ESM)}

Electronic Supplementary Material (ESM) associated with this article can be found at the website of CRC at http://www.akademiai.com/content/120427/

Electronic Supplementary Table S1. HMW-GS for bread making quality of wheat varieties in Pakistan

Electronic Supplementary Table S2. LMW-GS for bread making quality of wheat varieties in Pakistan

Electronic Supplementary Figure S1. Histogram for HMW-GS and LMW-GS in the experimental wheat genotypes

Electronic Supplementary Figure S2. Frequency of different alleles encoded by Glu-A1 (a), Glu-B1 (b) and Gu-D1 (c)

Electronic Supplementary Figure S3. Percentage of quality scores fixed in Pakistani germplasm

Electronic Supplementary Figure S4. Percent contribution of wheat genomes towards allelic variation of HMW-GS

Electronic Supplementary Figure S5. Frequency of different alleles encoded by Glu-A3 (a), Glu B3 (b) and Glu-D3 (c) 